\title{
Commentaries
}

\section{Host, heredity and helicobacter}

Helicobacter pylori is considered one of the most common pathogenic infections of mankind. Despite its worldwide distribution, the pathogenesis of $H$ pylori associated gastroduodenal disease remains poorly understood. What is clear is that only a minority of infected individuals develop severe inflammation leading to peptic ulcers or gastric cancer, the more severe manifestations of helicobacter infection. What are the factors which determine whether an infected individual will develop severe disease? It has been suggested that phenotypic or genotypic differences among bacterial isolates may be important in disease. ${ }^{1}$ There is evidence to suggest that individuals infected with strains of $H$ pylori which express the cytotoxin associated gene product CagA, a marker for the presence of a "pathogenicity island", are more likely to develop peptic ulcers or gastric cancer. ${ }^{2-5}$ Recent observations suggest that polymorphism of vacA genotypes may determine whether an $H$ pylori infected individual develops gastritis or an ulcer. ${ }^{6}$ However, only a small fraction of the estimated $60 \%$ of all individuals infected with such strains of $H$ pylori develop severe gastroduodenal disease (peptic ulcers or gastric cancer). This suggests that the host is also a factor in determining the ultimate clinical outcome following helicobacter infection.

In fact, several laboratories have provided evidence that the host response is an important determinant in helicobacter associated disease processes. Mouse models of disease are among the most amenable to genetic analysis and of the available mouse models, the $H$ pylori mouse model is limited by a delayed course of disease progression and consistently low inflammation scores. For instance, moderate to severe inflammation is present in $H$ pylori infected C57BL/6 mice only after six months or more. An alternative model of helicobacter disease is the Helicobacter felis mouse model which has been used extensively to examine how the host response prevents and/or exacerbates helicobacter induced gastroduodenal disease. ${ }^{7-11}$ In the mouse $H$ felis infection model, several inbred strains of mice, including $\mathrm{C} 57 \mathrm{BL} / 6, \mathrm{C} 3 \mathrm{H} / \mathrm{He}$, and SJL, exhibit severe inflammation/gastric atrophy ("high responder") after infection whereas other inbred strains, including $\mathrm{BALB} / \mathrm{c}, \mathrm{CBA} / \mathrm{Ca}$, and $\mathrm{C} 3 \mathrm{H} / \mathrm{HeJ}$, are low gastritis/atrophy responders to $H$ felis infection. These results suggest that the nature of the host immune or inflammatory response to $H$ pylori infection in humans may also be important in determining disease outcome.

The report by Sutton et al, in this issue (see page 335), represents an important next step in determining the basis for differential host response to helicobacter infections. These investigators used the $H$ felis model, and infected several high responder mouse strains as well as a low responder strain and the $\mathrm{F} 1$ crosses between the low responder and high responder strains. They then examined the degree of bacterial colonisation, several parameters of the gastric inflammatory response and serum anti- $H$ felis antibody responses in these populations of mice. For the most part, no significant differences in bacterial colonisation were observed. In contrast, both the low responder
CBA/Ca strain as well as the $\mathrm{F} 1$ progeny from high and low gastritis/atrophy responsive strains always exhibited low inflammation. Thus, low inflammation was a "dominant" response.

The results of the antibody responses from data reported here as well as elsewhere are more complicated. The parental low inflammation strain, $\mathrm{CBA} / \mathrm{Ca}$, also exhibited low serum antibody responses as did the $\mathrm{F} 1$ mice between $\mathrm{CBA} / \mathrm{Ca}$ and either $\mathrm{C} 3 \mathrm{H} / \mathrm{He}, \mathrm{C} 57 \mathrm{BL} / 6$, or SJL, three high inflammation parental mouse strains. This result alone could be consistent with a linkage of low antibody response to low atrophy/gastritis. However, although high inflammation $\mathrm{C} 3 \mathrm{H} / \mathrm{He}$ mice exhibited high levels of IgA, IgG1 and IgG2a serum antibodies, another high atrophy/gastritis parental mouse, the SJL strain, produced relatively low levels of serum IgA and IgG1 and has a genetic deletion of the IgG2a subclass. The other high atrophy/gastritis strain examined in this study, C57BL/6, produced high levels of serum IgA, low levels of serum IgG1 and is also unable to produce IgG2a. Furthermore, although the analysis was not as complete as reported by Sutton et al, another atrophy/gastritis unresponsive mouse strain, BALB/c, was previously reported by this group to produce moderate to high levels of $\mathrm{H}$ felis specific serum $\mathrm{IgG}^{8}$ Also, the low inflammation $\mathrm{C} 3 \mathrm{H} / \mathrm{HeJ}$ mouse strain produced equivalent or even slightly higher serum and salivary antibody levels than high inflammation $\mathrm{C} 3 \mathrm{H} / \mathrm{He}$ mice, ${ }^{10}$ a situation which may be further complicated by the lipopolysaccharide non-responsiveness of $\mathrm{C} 3 \mathrm{H} / \mathrm{HeJ}$ mice. Finally, local gastric IgA and IgG antibody production, which were not examined in this study, may be more relevant to gastric inflammation than serum antibodies.

Thus, although a state of low atrophy/gastritis in response to $H$ felis infection seems to be a dominant trait, the linkage of this phenomenon to antibody production seems to be less firm and much more complicated. This is an important point because the authors speculate, largely on the basis of low antibody responses in their $H$ felis infected F1 mice, that a dominant low response may actually represent active suppression of the host immune/ inflammatory response to helicobacter infections. Active suppression has been hypothesised by others to result in a state of low inflammatory/immune responsiveness in human $H$ pylori infections. Thus, although the studies reported by Sutton et al are not definitive in all respects, they do reveal the somewhat unexpected dominance of a low inflammatory response to $H$ felis infection. They also point out the usefulness of genetic quantitative trait analysis in probing the mechanisms of helicobacter associated diseases. Additional studies using similar methods have the potential to provide exciting new information on helicobacter-host interactions.

The authors laboratories are supported by grants DK-46461 and AI 40701 from the National Institutes of Health.

J G NEDRUD

Institute of Pathology,

Case Western Reserve University,

School of Medicine, Cleveland, Ohio, USA

S J CZINN

Department of Pediatrics,

Case Western Reserve University,

School of Medicine, Cleveland, Ohio, USA 
Correspondence to: Dr Czinn, Department of Pediatrics and Pathology, Case Western Reserve University, RB\&C Hospital, 11100 Euclid Avenue, Cleveland, Ohio 44106, USA

1 McGee DJ, Mobley HL. Mechanisms of Helicobacter pylori infection: bacterial factors. Curr Top Microbiol Immunol 1999;241:155-80.

2 Crabtree JE, Taylor JD, Wyatt JI, et al. Mucosal IgA recognition of Helicobacter pylori $120 \mathrm{kDa}$ protein, peptic ulceration, and gastric pathology. Lancet 1991;338:332-5.

3 Cover TL, Blaser MJ. Helicobacter pylori: A bacterial cause of gastritis, peptic ulcer disease, and gastric cancer. ASM News 1995;61:21-6.

4 Covacci A, Censini S, Bugnoli M, et al. Molecular characterization of the $120 \mathrm{kDa}$ immunodominant antigen of Helicobacter pylori associated with cytotoxicity and duodenal ulcer. Proc Natl Acad Sci USA 1993;90:5791-5. Xiang Z, Censini S, Bayeli PF, et al. Analysis of expresson of CagA and VacA virulence factors in 43 strains of Helicobacter pylori reveals that clinical solates can be divided into two major types and that CagA is not necessary for expression of the vacuolating cytotoxin. Infect Immun 1995;63:94-8.
6 Atherton JC, Peek R Jr, Tham KT, et al. Clinical and pathological importance of heterogeneity in vacA, the vacuolating cytotoxin gene of Helicobacter pylori. Gastroenterology 1997;112:92-9.

7 Mohammadi M, Redline R, Nedrud J, et al. Role of the host in pathogenesis of Helicobacter-associated gastritis: $\mathrm{H}$ felis infection of inbred and congenic mouse strains. Infect Immun 1996;64:238-45.

8 Sakagami T, Dixon M, O'Rourke J, et al. Atrophic gastric changes in both $\mathrm{H}$ felis and $\mathrm{H}$ pylori infected mice are host dependent and seperate from antral gastritis. Gut 1996;39:639-48.

9 Fox JG, Li X, Cahill RJ, et al. Hypertrophic gastropathy in Helicobacter felis-infected wild type C57BL/6 mice and p53 Hemizygous transgenic mice. Gastroenterology 1996;110:155-66.

10 Sakagami T, Vella J, Dixon MF, et al. The endotoxin of Helicobacter pylori is a modulator of host-dependent gastritis. Infect Immun 1997;65: 3310-16.

11 Wang TC, Goldenring JR, Dangler C, et al. Mice lacking secretory phospholipase A2 show altered apoptosis and differentiation with Helicobacter felis infection. Gastroenterology 1998;114:675-89.

\section{Glucocorticoid unresponsiveness in severe ulcerative colitis}

Although relatively little is known about the cellular and molecular immunopathology of ulcerative colitis, most would agree that it is an inflammatory disease. There is some support for the hypothesis that this inflammatory process is, at least in part, driven by $\mathrm{T}$ cells and their cytokine products. For example, anti-CD4 $\mathrm{T}$ cell monoclonal antibodies have been shown to abrogate disease in an animal "model" of colitis ${ }^{1}$. Anecdotally, monoclonal antibody against tumour necrosis factor $\alpha$ (derived in part from $\mathrm{T}$ cells) is effective therapy for ulcerative colitis. ${ }^{2}$ Finally, the fact that the powerful $\mathrm{T}$ cell suppressant cyclosporin $\mathrm{A}$ is effective in the treatment of acute severe colitis ${ }^{3}$ also suggests possible $\mathrm{T}$ cell involvement, although it should be borne in mind that cyclosporin A also has inhibitory effects on other inflammatory cells, including monocytes, basophils, and mast cells.

If this hypothesis were correct, one might expect that glucocorticoids, which are very potent inhibitors of $\mathrm{T}$ cell activation and cytokine secretion, would be an effective treatment for ulcerative colitis. Yet glucocorticoid therapy for acute exacerbations of ulcerative colitis frequently fails. A recent retrospective study ${ }^{4}$ of 97 patients with severe ulcerative colitis showed that, despite systemic glucocorticoid therapy, $34 \%$ required colectomy within 30 days of presentation. If, then, the "T cell hypothesis" of ulcerative colitis pathogenesis is not to be discarded completely, alternative explanations must be sought for this heterogeneity of clinical response.

One possibility is that severe ulcerative colitis is itself heterogeneous in terms of its pathology. The study cited above $^{4}$ suggested that patients failing to respond to glucocorticoid therapy were more likely to have fever, persistent diarrhoea, rectal bleeding, and elevated serum concentrations of C-reactive protein at three days after admission than those who did respond. Another study ${ }^{5}$ has suggested that patients with ulcerative colitis refractory to glucocorticoid therapy have a higher incidence of perinuclear staining anti-neutrophil cytoplasmic antibody than glucocorticoid sensitive patients and normal controls. Although the significance, if any, of these clinical phenomena is presently unknown, they suggest the possibility of heterogeneity in the pathogenesis of severe ulcerative colitis, which might in turn influence the clinical response to glucocorticoid therapy.

A second possibility, tackled in this issue by Hearing et al (see page 382), is that glucocorticoid refractory acute severe ulcerative colitis arises in patients whose $\mathrm{T}$ cells are relatively resistant to glucocorticoid inhibition. In this study, the authors classified 18 patients with acute severe ulcerative colitis according to their clinical response (at seven days after admission) to a standardised course of intravenous glucocorticoid therapy at high dosage as complete responders (three or fewer stools daily with no visible blood), partial responders (four or more stools daily or visible blood with no indication for colectomy), or treatment failures (colectomy indicated). Concentration response curves were constructed for the antiproliferative effect of glucocorticoids on lectin stimulated peripheral blood $\mathrm{T}$ cells from these patients obtained within 48 hours of admission. Proliferation of peripheral blood $T$ cells from five of the seven patients classified as partial responders or treatment failures was inhibited by less than $60 \%$ even at supra-physiological glucocorticoid concentrations. In contrast, proliferation of $\mathrm{T}$ cells from all 11 of the complete responders was inhibited by at least $60 \%$ at glucocorticoid concentrations that might be expected to be achieved in the peripheral blood in the course of intravenous glucocorticoid therapy at high dosage. When measured again three months later when the patients were in remission (some as a result of colectomy), these differences between the groups were no longer apparent, reflecting an increase in glucocorticoid sensitivity of $\mathrm{T}$ cells from the partial responder and treatment failure patients.

Similar correlations between $\mathrm{T}$ cell sensitivity to glucocorticoid inhibition and the clinical response to glucocorticoid therapy have been reported in other inflammatory diseases such as asthma (reviewed in ${ }^{6}$ ), rheumatoid arthritis and allograft rejection (cited by Hearing et al). These observations support a role for activated $\mathrm{T}$ cells in the pathogenesis of these diseases and also emphasise that, in a subset of patients with these diseases and likely others, glucocorticoid therapy is not viable because systemic glucocorticoid concentrations achieved even at high dosage are insufficient to inhibit $T$ cell function significantly and, by inference, disease progression.

The mechanism of this phenomenon has been best studied in glucocorticoid resistant asthmatics, whose disease fails to respond to glucocorticoid therapy at high dosage despite the fact that their airways obstruction is clearly reversible in response to inhaled $\beta 2$-agonists. These patients show no abnormalities of glucocorticoid absorption and clearance, and no innate abnormalities of the hypothalamus/pituitary/adrenal axis. ${ }^{6}$ Furthermore, they are not immune to developing the unwanted Cushingoid effects of glucocorticoid therapy. These observations suggest that the phenomenon of glucocorticoid resistance in these patients is not generalised, as in some rare cases of 
primary cortisol resistance, but somehow compartmentalised to $\mathrm{T}$ cells and possibly other inflammatory cells. It has been suggested that relative glucocorticoid resistance may be induced in $\mathrm{T}$ cells by the local inflammatory environment, as exposure of $\mathrm{T}$ cells to inflammatory cytokines in vitro reversibly increases their resistance to glucocorticoid inhibition, possibly at least partly by reducing the binding affinity of their intracellular glucocorticoid receptors for ligand. ${ }^{6}{ }^{7}$ This is not, however, an entirely satisfactory explanation as most patients with severe asthma do respond to glucocorticoid therapy, despite the fact that, presumably, their $\mathrm{T}$ cells are in a similar inflammatory environment to those who do not. Similarly, in the present study on ulcerative colitis, although the numbers of patients were small, disease severity at presentation did not predict the subsequent response to glucocorticoid therapy. Another proposed mechanism for glucocorticoid resistance in $\mathrm{T}$ cells is inappropriate, elevated expression of other secondary messenger proteins such as the transcriptional activator protein $\mathrm{AP}-1$ which can bind to, and inactivate the glucocorticoid receptor/ligand complex. ${ }^{8}$ Finally, these phenomena may be superimposed on a background of variable, innate $T$ cell glucocorticoid responsiveness which is known to be highly variable even in normal people. $^{9}$

The phenomenon of glucocorticoid resistance deserves further exploration in a wider variety of diseases, as its early recognition may save patients unnecessary and futile exposure to glucocorticoid therapy and its unwanted effects. Early assessment of possible glucocorticoid resistance may also facilitate management decisions in diseases such as acute, severe ulcerative colitis. Proliferation assays such as that described in the study by Hearing et al are relatively cheap and may be completed within 48 hours. Further studies are necessary better to define the repeatability and predictive value of these assays in larger groups of patients.

C J CORRIGAN

Clinical Senior Lecturer,

Honorary Consultant Physician,

Academic Department of Cardiopulmonary Medicine,

Imperial College School of Medicine,

Charing Cross Campus,

Fulham Palace Road,

London W6 8RF, UK

email: chris.corrigan@kcl.ac.uk

1 Hibi T, Iwao Y, Yajima T, et al. Immunosuppressive agents in the treatment of Crohn's disease and ulcerative colitis. F Gastroenterol 1995;30(suppl 8):121-3.

2 Evans RC, Clarke L, Heath P, et al. Treatment of ulcerative colitis with an engineered human anti-TNF alpha antibody CDP571. Aliment Pharmacol Ther 1997;11:1031-5.

3 Lichtiger S, Present DH, Kornbluth A, et al. Cyclosporine in severe ulcerative colitis refractory to steroid therapy. $N$ Engl f Med 1994;330:1841-5.

4 Lindgren SC, Flood LM, Kilander AF, et al. Early predictors of glucocorticosteroid treatment failure in severe and moderately severe attacks of ulcerative colitis. Eur f Gastroenterol Hepatol 1998;10:831-5.

5 Sandborn WJ, Landers CJ, Tremaine WJ, et al. Association of antineutrophil cytoplasmic antibodies with resistance to treatment of left-sided ulcerative colitis: results of a pilot study. Mayo Clinic Proc 1996;71:431-6.

6 Corrigan CJ. Glucocorticoid resistant asthma: T-lymphocyte defects. Am $\mathcal{F}$ Respir Crit Care Med 1996;154:S53-5.

7 Kam JC, Szefler SJ, Surs W, et al. Combination IL-2 and IL-4 reduces glucocorticoid receptor binding affinity and $\mathrm{T}$ cell response to glucocorticoids. f Immunol 1993;151:3460-6.

8 Adcock IM, Lane SJ, Brown CR, et al. Abnormal glucocorticoid receptor-activator protein 1 interaction in steroid-resistant asthma. $\mathcal{F}$ Exp Med 1995;182:1951-8.

9 Walker KB, Potter JM, House AK. Interleukin 2 synthesis in the presence of steroids: a model of steroid resistance. Clin Exp Immunol 1987;68:162-7.

\section{Mismatch repair deficit: a gain for diagnostic histopathology}

Cawkwell and colleagues, in this issue (see page 409), describe an immunohistochemical method which enables the status of a colorectal carcinoma with respect to the two mismatch repair (MMR) genes, hMLH1 and hMSH2, to be ascertained from paraffin wax sections produced by any diagnostic histopathology laboratory.

Despite many advances in the pathological management of colorectal cancer since Dukes and Bussey ${ }^{1}$-for example, taking account of the number of lymph nodes containing metastatic tumour or the nature of the infiltrating margin of the tumour, ${ }^{2}$ staging systems have not hitherto provided precise information about the biology and likely behaviour of the tumour in individual cases. By adopting the relatively simple methodology proposed by Cawkwell and colleagues, it should now be possible to assign patients reliably to one of the two main specific categories of MMR status, thereby identifying individuals who are likely to have the hereditary non-polyposis colon cancer (HNPCC), Lynch syndrome, genotype. Although there is a theoretical possibility that HNPCC could be due to a defect at one of the other MMR genes (e.g. PMS1, PMS2, hMSH6/GTBP, and hMSH3), all patients with HNPCC so far documented have a deficit at either MLH1 or MSH2.

This is a major advance. At a stroke, a small but significant group of patients with a relatively good prognosis but at risk of developing metachronous colorectal and other carcinomas can now be identified. It should be noted that this does not just mean the 2 or $3 \%$ of patients with colorectal cancer who are genetically HNPCC family members. Up to $13 \%$ of sporadic colorectal cancers also display a MMR deficient phenotype, ${ }^{3}$ which, as in HNPCC, is both associated with a better prognosis ${ }^{45}$ and is a marker for increased risk of development of metachronous colorectal cancers. It is also reported that patients with such "sporadic" replication error (RER) positive colorectal cancers may have, after all, a family history of colorectal cancer. $^{4}$

Importantly, it has also been shown that RER positive colorectal cancers, both familial and "sporadic", are relatively resistant to chemotherapy ${ }^{6}$ and, by implication, radiotherapy. It will now be possible to identify such patients in advance of therapy and plan treatment accordingly.

Perhaps the most valuable application of this immunohistochemical test will be in the large group of patients who, because of their family history, are worried about their mutator phenotype status. Hitherto, it has been necessary to undertake specific tests on DNA from peripheral blood or specimens of tumour, testing for microsatellite instability using relatively inaccessible and therefore more expensive PCR technology. ${ }^{78}$ This has presented practical obstacles and, in order to keep numbers manageable, it has been necessary to restrict the availability of genetic testing to those with a family history suggestive of HNPCC. For the reasons outlined earlier, it has become clear that a good case can be made for all colorectal cancers to be screened for MMR deficiency. Use of this technology will not only add power to the geneticist's elbow, but will permit the identification of an important subgroup of patients in the everyday practice of colorectal surgery. In the case of 
apparently sporadic colorectal cancers, in which the average age is 63 years and there is no strong family history, MMR deficient status will tell us only about that particular patient and no further genetic testing is indicated. However, when there are other factors such as young age or family history in a patient with a tumour lacking hMLH1 or hMSH2 protein, specific genetic studies are indicated, as a means of initiating an investigation of the whole family for cancer prevention. The use of a preliminary test such as this for screening would cast the net widely for MMR defective tumours, while keeping the workload of genetic laboratories manageable.

The general adoption of this technology would also provide a rationale for a more economical use of resources for follow up of patients after surgery, by focusing on those patients at risk of developing a metachronous cancer. The authors suggest that those with RER negative tumours could be discharged from follow up after three years. This would provide a rational basis for a policy which some surgeons have been following rather pragmatically for some time.
Academic Department of Pathology,

St Mark's Hospital,

Northwick Park,

Watford Road,

Harrow HA1 3UF, UK

1 Dukes CE, Bussey HJR. The spread of rectal cancer and its effect on prognosis. Br f Cancer 1958;12:309-20.

2 Jass JR, Love SB, Northover JMA. A new prognostic classification of rectal cancer. Lancet 1987;i:1303-6.

$3 \mathrm{Kim} \mathrm{H}$, Jen J, Vogelstein B, et al. Clinical and pathological characteristics of sporadic colorectal carcinomas with DNA replication errors in microsatellite sequences. Am F Pathol 1994;145:148-56.

4 Lothe RA, Peltomaki P, Meling GI, et al. Genomic instability in colorectal cancer: relationship to clinicopathological variables and family history. Cancer Res 1993;53:5849-52.

5 Bubb VJ, Curtis LJ, Cunningham C, et al. Microsatellite instability and the role of hMSH2 in sporadic colorectal cancer. Oncogene 1996;12:2641-9.

6 Boland CR. Roles of the DNA mismatch repair genes in colorectal tumorigenesis [review]. Int $\mathcal{F}$ Cancer 1996;69:47-9.

7 Dietmaier W, Wallinger S, Bocker $\mathrm{T}$, et al. Diagnostic microsatellite instability: definition and correlation with mismatch repair protein expression. Cancer Res 1997;57:4749-56.

8 Cawkwell L, Quirke P. A new class of colorectal cancer gene. Gut 1995;36: 641-3.
Over the past 15 years, opinion regarding the suitability of alcoholic patients for liver transplantation has fluctuated from overt disapproval to optimism that liver transplantation might have a beneficial effect on alcoholic behaviour. The former view was evaluated by an opinion survey among the general population, general practitioners and hospital specialists in Great Britain, in which the vignette of an alcoholic in need of liver transplant was consistently rated as justifying a low priority. ${ }^{1}$ The origin of optimism about the salutary effects of transplantation on alcoholic relapse came from Thomas Starzl, who coined the aphorism that, "liver transplantation was the ultimate sobering experience". ${ }^{2}$ The current status of liver transplantation for alcoholic liver disease rests somewhere between these two extremes.

Alcoholic liver disease is a common diagnosis among patients selected for liver transplantation in North America and Europe. It often occurs in conjunction with chronic hepatitis $\mathrm{C}$ infection. All transplant programmes try to identify patients who will remain abstinent, although how best to achieve this end is less certain. The study by Pageaux et al, in this issue (see page 421), is unusual in not including psychiatry as part of the pretransplant assessment. ${ }^{4}$ The requirement for a fixed period of abstinence, the so called six month rule, as a predictor of future abstinence is in widespread use among transplant centres in North America and Europe. ${ }^{4}$ Many addiction specialists remain skeptical about the usefulness of the six month rule, both because it ignores the complexity of addictive behaviour, and because it does not successfully predict future drinking by alcoholic candidates for liver transplantation. ${ }^{5-8}$

Initial patient and graft survival for alcoholic patients after transplantation is similar to that in patients transplanted for other forms of chronic liver diseases. ${ }^{39}$ Quality of life measures, such as rate of returning to work, are similar in alcoholic and non-alcoholic liver transplant recipients. However, data from one of the few prospective stud- ies show that patient survival curves decline more rapidly after two years in alcoholic recipients compared with non-alcoholics. ${ }^{9}$ Given that the rate of retransplantation is lower in alcoholics than in non-alcoholic recipients, these data may reflect a greater reluctance on the part of transplant programmes to retransplant alcoholics.

The greatest area of controversy concerns alcoholic relapse after liver transplantation. This topic is confounded by difficulty in determining accurate data on drinking behaviour, and a more fundamental conflict on the meaning of alcohol relapse when it occurs in alcoholics. Addiction specialists and transplanters use different interpretations of relapse into alcohol use. Addiction specialists eschew the term recidivism with its connotations of blame, and refer to minor, isolated drinking episodes as slips. ${ }^{5}$ Indeed, a slip may serve a useful purpose by indicating to the alcoholic that they need more support or treatment for their alcoholism. Conversely, liver transplanters tend to regard any use of alcohol as a failure of compliance, which should have serious consequences, such as removal from the waiting list. These dichotomous views on the nature of alcoholism were highlighted by a conference on Liver Transplantation in Alcoholics held under the auspices of the National Institutes of Health in 1997 which served to bring clinical researchers in liver transplantation and addiction medicine together for the first time..$^{3-5} 9$

Despite difficulty in getting robust data and differences in accepting a model of addiction which distinguishes slips (minor, isolated drinking) from relapses (prolonged and harmful drinking), liver transplanters and addiction specialists are finding common ground in the consistent results regarding alcohol use which are coming to light. In this issue Pageaux et al present a retrospective data analysis regarding alcohol use and other parameters of clinical well-being in 54 alcoholic liver transplant recipients. Their data are broadly consistent with at least 17 other published reports on the outcome of liver transplantation in alcoholic patients. ${ }^{10}$ Typically, roughly one third to one half of alcoholic liver transplant recipients will report some use of alcohol in the first five years after transplantation. Although many studies suggest that the consequences of alcohol use are minimal for many recipients, perhaps because alcohol is consumed in small amounts on infrequent occasions, there 
are few good data to be certain. Conversely, between 10 and $20 \%$ of alcoholic liver transplant recipients will drink excessively, yet over five years, few of these patients develop significant liver injury. The major deleterious effects of heavy alcohol use in the first five years after liver transplantation are on extrahepatic health, thereby emphasising that the injurious effects of alcohol are multisystemic. ${ }^{78}$

What do these observations teach us about alcoholism and the urge to drink excessively? Like the cliche about the glass being half full or half empty, the data on alcohol relapse after liver transplantation can be viewed as surprisingly good or disappointingly bad. Compared with the outcome of other forms of intervention against alcoholism, a rate of sobriety of $50 \%$ at five years is at least as good as most alcoholism treatment programmes. Indeed, such data may overestimate significant drinking, as some of the patients who are classified as relapsers report occasional slips which would not be considered treatment failures in today's standard alcoholism literature. However, it is noteworthy that many alcoholic patients return to alcohol use after liver transplantation despite the devastating effects of alcohol on their lives, and despite evidence of continuing alcoholic injury. Our goal should be to reduce further the frequency of relapses to excessive, harmful drinking. In order to achieve this goal, it will be necessary to understand the factors that promote or inhibit a return to drinking, and how relapsers contrast with those who establish long term sobriety. It is naive to presume that paradigms drawn from alcoholism treatment programmes will translate directly into the liver transplant population. Alcoholic liver transplant candidates are less likely to consider themselves in need of treatment for alcoholism than matched controls in an alcoholism treatment programme. ${ }^{11}$ Similarly, after transplantation, alcoholic patients frequently lack motivation for alcoholism treatment. Enhancing the sense of commitment to treatment may be an important compo- nent in improving control of alcoholism in the $50 \%$ of recipients in danger of relapse in the first five years after liver transplantation. Although much has been learnt about alcoholics and alcoholism from retrospective audits of transplant databases, future advances will require hypothesis testing by means of longitudinal, prospective studies.

M R LUCEY R M WEINRIEB

Departments of Medicine and Psychiatry,

The University of Pennsylvania School of Medicine,

Philadelphia, Pennsylvania, USA

Correspondence to: Michael R Lucey, MD, Associate Professor of Medicine, Director of Hepatology, Medical Director of the Liver Transplantation Programme, Division of Gastroenterology, Department of Medicine, Hospital of the University of Pennsylvania, 3400 and Spruce, Philadelphia, PA 19104, USA (email: lucey@mail.med.upenn.edu).

1 Neuberger J, Adams D, MacMaster P, et al. Assessing priorities for allocation of donor liver grafts: survey of public and clinicians. BMF 1998;317:172-5.

2 Starzl TE, Van Thiel D, Tzakis AG, et al. Orthotopic liver transplantation for alcoholic cirrhosis. fAMA 1988;260:2542-4.

3 Belle SH, Beringer KC, Detre KM. Liver transplantation for alcoholic liver disease in the United States: 1988 to 1995. Liver Transplantation and disease in the United

4 Everhart JE, Beresford TP. Liver transplantation for alcoholic liver disease: a survey of transplantation programs in the United States. Liver Transplantation and Surgery 1997;3:220-6.

5 Fuller RK. Definition and diagnosis of relapse to drinking. Liver Transplantation and Surgery 1997;3:258-62.

6 Yates WR, Martin M, LaBrecque D, et al. A model to examine the validity of the 6-month abstinence criterion for liver transplantation. Alcohol Clin Exp Res 1998;22:513-17.

7 Lucey MR, Carr K, Beresford TP, et al. Alcohol use after liver transplantation in alcoholics - a clinical-cohort follow-up study. Hepatology 1997;25: 1223-7.

8 Tang H, Boulton R, Gunson B, et al. Patterns of alcohol consumption after liver transplantation. Gut 1998;43:140-5.

9 Wiesner RH, Lombardero M, Lake JR, et al. Liver transplantation for endstage alcoholic liver disease: an assessment of outcomes. Liver Transplantation and Surgery 1997;3:231-9.

10 Lucey MR. Liver transplantation for alcoholic liver disease: a progress report. Graft 1999;2:573-9.

11 Weinrieb R, Lucey $M$, Van Horn D, et al. Drinking behavior and drug use in alcoholic liver transplant candidates: a comparison with alcoholics in a treatment unit. Presentation at the AST Meeting, Chicago, Ill. May, 1999.

\section{Transplantation in HCV: whither immunosuppression}

Cirrhosis due to hepatitis C (HCV) is now the most common indication for liver transplantation in western Europe and the United States. Given the absence of effective prophylaxis, recurrent HCV infection is almost inevitable. Although the natural history and outcome of recurrent HCV infection are now better documented, the factors which influence the recurrence of hepatitis and its subsequent progression are still unclear. ${ }^{1}$ In fact, the variation in the consequences of liver disease associated with recurrent $\mathrm{HCV}$ infection suggest that several viral and host factors are important in determining clinical outcome. Despite the rapid growth in knowledge of the molecular biology of hepatitis $\mathrm{C}$, our understanding of the immunopathology of chronic HCV infection is still poor. Immunosuppression may well be the single most important factor responsible for variations in clinical outcome. Evidence of its potential involvement includes a more aggressive histological picture in non-transplanted patients with congenital or acquired immunodeficiency ${ }^{2}{ }^{3}$ as well as among liver transplant recipients who require treatment with steroid boluses for acute rejection. ${ }^{4}$ In the transplant setting, however, there are conflicting reports of the effects of adjuvant immunosuppression on the natural history of $\mathrm{HCV}$ recurrence.

In this issue (see page 427) Papatheoridis and collaborators present their results on the impact of different immunosuppressive regimens on longitudinal variation in $\mathrm{HCV}$ viraemia. This non-randomised study examined fluctuations in viraemia, including the early course of $\mathrm{HCV}$ infection, in a cohort of $47 \mathrm{HCV}$ transplant recipients. Although the patients were not randomised to the different immunosuppressive regimens, the type of treatment was not selected on the basis of patient characteristics, but changed according to different study protocols in use at the centre over time.

The authors found that the level of HCV RNA three months after transplantation is higher in patients treated with a single drug regimen as the initial immunosuppressive therapy. However, at 12 months HCV RNA levels correlated only with duration of steroid treatment, and no significant difference was observed between patients treated with either cyclosporin or tacrolimus as monotherapy. The authors did find a strong correlation between the HCV RNA level 12 months after liver transplantation and the fibrosis score at this time. Furthermore, the extent of fibrosis was also associated with the occurrence of an acute hepatitis episode.

Despite being of potential interest, the findings from this small study are preliminary as there remain many issues requiring clarification. The impact of steroids on $\mathrm{HCV}$ 
replication following liver transplantation should be further documented. Steroids have been shown to increase the level of HCV viraemia in other studies, both in transplant and non-transplant patients. However, the exact mechanism is unknown and the data presented suggest that direct stimulation of viral replication is unlikely. In fact, the HCV RNA level did not differ after 1-2 weeks between patients treated with and without steroids and at three months it was significantly higher in patients treated without steroids. It is possible that steroid withdrawal may trigger an immunological attack with increased lysis of infected hepatocytes and subsequent release of virions into the circulation. In addition, controlled trials with a longer follow up will be required to assess the benefit of early steroid tapering after transplantation. In Europe, a large randomised, controlled trial is currently underway with a tacrolimus based regimen, including rapid steroid tapering after transplantation, to assess its effects on the recurrence of HCV one year post-transplantation. Although one group observed that a tacrolimus based regimen was associated with a significant reduction in survival in HCV transplant recipients, ${ }^{5}$ other studies failed to find a difference between the cyclosporin and tacrolimus based regimens in either the incidence or severity of recurrent hepatitis on the graft. ${ }^{67}$ In our series of 120 patients transplanted for HCV, we observed that the tacrolimus based regimen delayed recurrent hepatitis after liver transplantation but that $\mathrm{HCV}$ RNA levels were similar in patients treated with cyclosporin or tacrolimus. Further studies are therefore needed to establish whether tacrolimus based immunosuppression changes the natural history of HCV recurrence after liver transplantation. It may be possible to clarify the effect of tacrolimus or cyclosporin on HCV replication after transplantation using quantitative methods to measure HCV in serum and liver.

Papatheoridis and colleagues found a strong correlation between fibrosis and HCV RNA levels at 12 months. The HCV RNA level may therefore be a predictive factor of long term outcome after post-transplant HCV infection if it is confirmed that progression of fibrosis is the most important predictor of chronic hepatitis after transplantation.

It has been reported recently that severe initial recurrent hepatitis may be associated with the subsequent develop- ment of cirrhosis. ${ }^{8}$ In this study, the authors found a correlation between the extent of fibrosis at 12 months and a history of previous acute hepatitis, but not with its severity. Larger numbers of patients, longer follow up and a standard definition of severity of acute hepatitis should all contribute to a better understanding of the effect on outcome of early recurrent hepatitis after transplantation. If the occurrence of symptomatic acute hepatitis does influence the histological outcome, then early antiviral treatment may be beneficial.

In conclusion, although the cumulative five year survival of $\mathrm{HCV}$ positive recipients seems to be similar to that of liver transplant patients without $\mathrm{HCV}$ infection, the large number of patients with progressive $\mathrm{HCV}$ related graft damage suggests that a longer follow up period may impact negatively both on graft and patient survival. The management of hepatitis $\mathrm{C}$ after liver transplantation has therefore become a very important issue, as has the composition of the optimal immunosuppressive regimen.

T BIZOLLON C TREPO

Liver Unit,

Hôtel-Dieu,

Lyon 69288 Cédex 02,

France

1 Bizollon T, Mutimer D, Ducerf C, et al. Hepatitis C virus recurrence after liver transplantation. Gut 1999;44:575-8.

2 Bjoro K, Froland S, Yun Z, et al. Hepatitis C infection in patients with primary hypogammaglobulinemia after treatment with contaminated immune globulin. N Engl f Med 1994;331:1607-11.

3 Martin P, DiBiscegli AM, Kassianides C, et al. Rapidly progressive non-A, non-B hepatitis in patients with human immunodeficiency virus infection. Gastroenterology 1989;97:159-61.

4 Sheiner PA, Schwartz ME, Mor E, et al. Severe or multiple rejection episodes are associated with early recurrence of hepatitis $\mathrm{C}$ after orthotopic liver transplantation. Hepatology 1995;21:30-4

5 Mulligan DC, Goldtein RM, Levy MF. Hepatitis C virus infection in liver transplantation: effect of cyclosporine versus KK506 [abstract]. Chicago: transplantation: effect of cyclosporine versus KK

6 Flamm SL, Marcos A, Jenkins RL, et al. Tacrolimus is associated with decreased survival in patients undergoing transplantation with chronic hepatitis C infection [abstract]. Chicago: American Society of Transplant Physicians, 1996

7 Gane EJ, Portmann BC, Naoumov NV, et al. Long-term outcome of hepatitis infection after liver transplantation. $N$ Eng $\mathcal{F}$ Med 1996;334:821-7.

8 Rosen HR, Gretch DR, Oehkle M, et al. Timing and severity of initial hepatitis C recurrence as predictors of long-term liver allograft injury. Transplantation 1998;65:1178-82.
In this issue Lenzi et al (see page 435) provide convincing evidence that non-organ specific autoantibodies (NOSA) are highly prevalent in subjects exposed to hepatitis $\mathrm{C}$ virus (HCV), and their presence is associated with clinical and biochemical signs of liver disease. The observation that autoimmunity and HCV infection are associated is not new: several hundred hits are returned when Medline is interrogated using $\mathrm{HCV}$ and autoimmune, including autoantibodies, as coordinates. Invariably, however, the infected individuals are patients referred to hospital and who have undergone some form of selection, primarily dictated by the severity of their underlying disease. The population studied by Lenzi et al makes their paper unique.

During the screening of an unselected population of 7000 (the Dionysos study ${ }^{1}$ ) for indicators of liver disease,
See article on page 435

226 were positive for HCV markers. Lenzi and colleagues found NOSA in $25 \%$ of these subjects, a prevalence much higher than that of the 226 demographically matched uninfected individuals and of the $78 \mathrm{HBsAg}$ positive subjects from the Dionysos study. Within the HCV positive group, the presence of NOSA was significantly associated with clinical and biochemical evidence of liver disease.

The already high NOSA prevalence of $25 \%$ is certainly an underestimate as the serum samples were tested at a dilution of 1 in 40, and not at the conventional screening dilution of 1 in 10. Thus in a study investigating NOSA in a nosocomial paediatric HCV series, Gregorio and colleagues $^{2}$ found that the autoantibody prevalence more than doubled (from 27 to $65 \%$ ) if the samples were tested at a 1 in 10 dilution instead of 1 in 20 . What does the presence of NOSA in HCV infection mean? Do they carry a pathogenic or a clinical message?

The fact that in an unselected HCV positive population the presence of NOSA is associated with clinical manifestations of chronic liver disease and with high activities of 
alanine aminotransferase indicates incontrovertibly that there is a connection between autoantibodies and liver damage. Are NOSA also the cause of the damage? I concur with Lenzi et al that currently there are insufficient data to provide an answer. Does measurement of NOSA add anything to the information obtained through the conventional assessment of liver function? The number of reports describing adverse reactions to interferon treatment in autoantibody positive HCV patients is increasing steadily ${ }^{2-4}$ and invariably the autoantibody associated with these reactions is liver/kidney microsomal antibody type 1 (LKM-1). In addition to being the hallmark of the eponymous form of autoimmune hepatitis, LKM-1 is also present in up to $10 \%$ of chronically infected HCV patients. HCV/LKM-1 positive patients on interferon experience increases in aminotransferase activities, ${ }^{2-4}$ occasionally of such magnitude to warrant suspension of treatment, ${ }^{24}$ or may even, rarely, progress to LKM-1 positive autoimmune hepatitis. The amino acid sequence similarities between cytochrome P4502D6, the molecular target of LKM-1, and HCV polyprotein suggests that a process of molecular mimicry and crossreactive immunity is the link between viral and autoimmune condition. Whether, and at what stage, interferon treatment should be replaced by immunosuppression in LKM-1/HCV positive patients ${ }^{5}$ is still a matter of conjecture.

From the recent literature it is clear that LKM-1 should be measured in all patients with $\mathrm{HCV}$, especially those undergoing interferon treatment, and this should be done at a dilution of 1 in 10 . In the study by Gregorio and colleagues $^{2}$ treatment with interferon had to be suspended in four patients because of a notable rise in aminotransferse activities: three were LKM-1 positive, two at a titre of $1 / 10$. In that series the only other LKM-1 positive patient taking interferon had normal aminotransferases. LKM-1 seems to act as a danger signal whatever its titre. Interestingly, Cassani and colleagues ${ }^{6}$ have shown that the presence of LKM-1 in HCV infection is associated with a greater histological severity of liver disease and the recent localisation of CYP2D6 to the liver cell membrane-an ideal target for an autoantibody mediated attack-may explain why LKM-1 is associated with liver damage.

Methodologically, it should be noted that LKM-1 is notoriously difficult to identify and is frequently mistaken for antimitochondrial antibody in view of their similar immunofluorescence patterns on rodent renal tubules. ${ }^{7}$ This problem should hopefully be overcome in the near future when objective assays based on molecularly defined targets (i.e. CYP2D6) will replace the subjective immunofluorescence testing. Such assays are already available for research purposes, ${ }^{7-9}$ and commercial ones are being marketed and their performance assessed.

Autoimmunity in $\mathrm{HCV}$ infection is not limited to autoantibody seropositivity but embraces the full spectrum of textbook autoimmune disorders, ${ }^{10}$ ranging from the non-organ specific cryoglobulinaemia to the exquisitely organ specific autoimmune thyroiditis and includes such conditions as rheumatoid arthritis, Sjögren's syndrome, glomerulonephritis, lichen planus, periarteritis nodosa, and type 1 diabetes. At the opposite extreme of HCV associated autoimmune disease, the pathogenic mechanisms differ. Thus in cryoglobulinaemia and HCV associated glomerulonephritis it is the formation of immune complexes that causes pathology. Hepatitis $\mathrm{C}$ viral constituents are integral components of the immune complex and interferon induced control of the viral infection is associated with an improvement in cryoglobulinaemia and glomerulonephritis. Interferon is also involved at the other end of the spectrum, but this time in unmasking subclinical or inducing de novo autoimmune thyroiditis. Appropriately, it is further work by Lenzi et al, only published in abstract form, that shows, firstly, the much more frequent appearance of thyroiditis in HCV patients who receive interferon if they also are positive for LKM-1 and, secondly, that the possession of the "autoimmune" haplotype HLA B8, DR3 is key to the autoimmune manifestations of $\mathrm{HCV}$ infection.

Professor of Immunology,

D VERGANI

Institute of Hepatology,

Royal Free and University College Medical School,

Gower Street Campus,

Harold Samuel House,

69-75 Chenies Merus,

London WC1E 6HX, UK

Email:D.Vergani@ucl.ac.uk

1 Bellentani S, Tiribelli C, Saccoccio G, et al. Prevalence of chronic liver disease in the general population of northern Italy: the Dionysos Study. Hepatology 1994;20:1442-9.

2 Gregorio GV, Pensati P, Iorio R, et al. Autoantibody prevalence in children with liver disease due to chronic hepatitis $\mathrm{C}$ virus (HCV) infection. Clin Exp Immunol 1998;112:471-6.

3 Muratori L, Zauli D, Giostra F, et al. LKM1 appearance in a HLA-DR3+ patient with chronic hepatitis $\mathrm{C}$ during interferon treatment. $f$ Hepatol 1993;18:259-60.

4 Dalekos GN, Wedemeyer H, Obermayer-Straub P, et al. Epitope mapping of cytochrome P4502D6 autoantigen in patients with chronic hepatitis C during alpha-interferon treatment . F Hepatol 1999;30:366-75.

5 Vergani D, Mieli-Vergani G. Type II autoimmune hepatitis. What is the role of the hepatitis C virus? Gastroenterology 1993;104:1870-3.

6 Cassani F, Cataleta M, Valentini P, et al. Serum autoantibodies in chronic hepatitis C: comparison with autoimmune hepatitis and impact on the disease profile. Hepatology 1997;26:561-6.

7 Kerkar N, Ma Y, Hussain M, et al. A novel assay for detecting antibodies to cytochrome P4502D6, the molecular target of liver kidney microsomal antibody type 1. F Immunol Methods 1999;223:227-35.

$8 \mathrm{Ma} \mathrm{Y}$, Gregorio G, Gaken J, et al. Establishment of a novel radioligand assay using eukaryotically expressed cytochrome P4502D6 for the measurement of liver kidney microsomal type 1 antibody in patients with autoimmune hepatitis and hepatitis C virus infection. F Hepatol 1997;26:1396-402.

9 Yamamoto AM, Johanet C, Duclos-Vallee JC, et al. A new approach to cytochrome CYP2D6 antibody detection in autoimmune hepatitis type-2 (AIH-2) and chronic hepatitis C virus (HCV) infection: a sensitive and quantitative radioligand assay. Clin Exp Immunol 1997;108:396-400.

10 Peakman M. Vergani D. Basic and clinical immunology. 1st edn. New York: Churchill Livingstone, 1997.
The embryology of the portal venous system is extremely complex and yet major congenital malformations of the system have been rarely described. However, the increased use of sophisticated ultrasound techniques and the increase in liver transplantation have resulted in an increase in the number of reports of these abnormalities during the past 15 years. There is also an increased awareness of associated complications from these abnormalities including late onset encephalopathy.

The systemic venous system develops from the intraembryonic anterior and posterior cardinal veins, whereas the portal venous system develops from the extraembryonic vitelline and umbilical veins which provide drainage from the yolk sac and placenta, respectively. ${ }^{1}$ The vitelline veins are paired vessels which drain into the primitive sinus venosus and by the end of the fourth week 
of gestation, three cross communications have developed between them in subhepatic, retroduodenal and subdiaphragmatic positions. ${ }^{2}$

The portal vein in the embryo is derived from the inferior section of the left vitelline vein, the retroduodenal communication, and the superior section of the right vitelline vein, and so forms the S-shaped configuration of the mature portal vein. The inferior vena cava is also formed from the amalgamation of several venous channels in the embryo including the right end of the sinus venosus and the right end of the subdiaphragmatic anastomosis of the vitelline veins. Early in development blood from the left umbilical vein passes through the subhepatic anastomosis and the ductus venosus to reach the retrohepatic portion of the inferior vena cava, the common hepatic vein in the embryo. Anatomically, the ductus venosus therefore forms a direct connection between the umbilical vein and the inferior vena cava and provides a functional bypass through which part of the oxygenated umbilical blood from the placenta flows directly into the inferior vena cava. ${ }^{3}$ Human fetal studies clearly demonstrate that the umbilical vein gives branches to the left segments of the liver and that the opening of the ductus venosus lies opposite the junction of the umbilical vein with the left branch of the portal vein. Roughly 40 to $50 \%$ of umbilical vein blood passes through the ductus venosus whereas the remainder flows through the liver sinusoids. ${ }^{3}$

The wall of the ductus venosus contains only a small amount of smooth muscle and no muscular sphincter. Postnatal closure of the channel commences during the first minute after birth but is not completed until days 15 to 20. The mechanism of closure is not clear but it may be related to a decrease in pressure within the portal venous system after cessation of umbilical vein flow. ${ }^{3}$

Congenital malformations of the portal venous system are unusual. A preduodenal position of the portal vein is the result of a persistence of the most caudal of the intervitelline anastomoses and this abnormality is found most frequently in association with biliary atresia, duodenal atresia, and annular pancreas. Persistence of both vitelline veins may result in portal vein duplication, whereas more complex defects may include portal drainage into the superior vena cava, right atrium, or azygos system of veins. ${ }^{4}$

Abernethy $(1793)^{5}$ gave the first description of a congenital extrahepatic mesenterico-caval anastomosis in association with an absence, or atresia, of the portal vein. The report was based on a postmortem examination of a 10 month old female infant who died of unknown cause. In addition to transposition of the great vessels and polysplenia, the portal vein terminated in the inferior vena cava at the level of the insertion of the renal veins. At least 13 examples of this type of congenital portosystemic anastomosis have now been reported, all in females. Complications in these cases included encephalopathy from the age of 44 years in one patient and a variety of liver tumours in five others which included focal nodular hyperplasia, adenoma, hepatoblastoma, and hepatoma. Recently it has been suggested that this type of congenital end-to-side portosystemic shunt should be designated as a "type 1" lesion. ${ }^{6}$

A second variety of side-to-side congenital extrahepatic portosystemic shunt ("type 2") has also been recognised in two adult and four paediatric patients, all of whom were males. ${ }^{6}$ This type of communication between the portal vein and the retrohepatic vena cava is generally believed to represent a persistence of the ductus venosus and is amenable to surgical division and restoration of a normal intrahepatic flow of portal blood. Two adult patients, aged 49 and 67 years, presented with the clinical and biochemi- cal features of hepatic encephalopathy. Surgical division of the shunt in the first patient resulted in severe portal hypertension secondary to intrahepatic portal vein hypoplasia and a portosystemic anastomosis was re-established as an emergency. ${ }^{7}$ The second patient responded well to surgical division of the shunt and there were no further episodes of encephalopathy. ${ }^{8}$ Surgical closures of "type 2 " side-to-side shunts were also performed in two children without complication. ${ }^{6}$ Congenital portosystemic shunts, analogous to those described in humans, are well recognised in a variety of animals including dogs and cats. ${ }^{9}$ Encephalopathic ataxia in these animals is followed by coma and the clinical signs are accompanied by increasing blood ammonia concentrations. Urolithiasis was also found in $53 \%$ of the animals and was believed to be secondary to urinary excretion of ammonia and urate.

A familial incidence of patent ductus venosus ("type 2" shunt) was described in three young brothers who presented with encephalopathy. The symptoms and biochemical changes were successfully reversed in the two who underwent surgical closure of the shunt. ${ }^{10} \mathrm{Jacob}$ et al, in this issue (see page 442), describe another family of three brothers in whom congenital "type 2 " side-to-side shunts were demonstrated with ultrasound, computed tomography and angiographic imaging. Two of the patients had evidence of mild encephalopathy, one of whom had bilateral renal stones. Two liver masses of focal nodular hyperplasia were detected in the third patient. The clinical observations of encephalopathy, liver tumour and male dominance in the two reports of familial "type 2 " shunts are therefore in accord with the previous single case reports. The authors of the current report suggest that their observations are in keeping with the transmission of an autosomal recessive trait.

In conclusion, reports of congenital portosystemic shunts have become more frequent during the past 15 years and they should be considered in the differential diagnosis of unexplained encephalopathy, particularly when symptoms appear in younger patients. The association of congenital shunts with primary liver tumours also suggests that a complete investigation of liver masses in young people should include a search for any congenital abnormality of the portal venous system. Surgical correction of "type 2" shunts prevents the onset of encephalopathy and can reverse established signs. Liver tumours can be resected safely, even in patients with "type 1 " shunts, ${ }^{2}$ but severe encephalopathy in "type 1 " lesions, which are associated with an absent intrahepatic portal vein, can only be relieved by liver transplantation.

E R HOWARD

Department of Surgery,

King's College Hospital,

Denmark Hill,

London SE5 9RS, UK

1 Dickson AD.The development of the ductus venosus in man and the goat. $\mathcal{F}$ Anat 1957;91:358-68.

2 Joyce AD, Howard ER. Rare congenital anomaly of the portal vein. Br F Surg $1988 ; 75: 1038-9$

3 Meyer WW, Lind J. The ductus venosus and the mechanism of its closure. Arch Dis Child 1966;41:597-605.

4 Marks C. Surgical implications of portal venous system malformation. Ann $R$ Coll Surg Eng 1974;55:299-306.

5 Abernethy J. Account of two instances of uncommon formation, in the viscera of the human body. Phil Trans R Soc 1793;83:59-66.

6 Howard ER, Davenport M. Congenital extrahepatic portocaval shunts-the Abernethy malformation. F Pediatr Surg 1997;32:494-7.

Abernethy malformation. F Pediatr Surg 1997,32.494-7. Barsky MF, Rankin RN, Wall WJ, et al. Patent ductus venosus
assessment and management. Can F Surg 1989;32:271-5.

assessment and management. Can $\mathcal{F}$ Surg 1989;32:271-5. 8 Kerlan RK, Sollenberger RD, Palubinskas AJ, et al. Portal-systemic encephalopathy due to a congenital portocaval shunt. AfR Am f Roentgenol 1982;139:1013-15.

9 Rothuizen J, Van den Ingh TS, Voorhout G, et al. Congenital porto-systemic shunts in sixteen dogs and three cats. $\mathcal{F}$ Small Anim Pract 1982;23:67-81.

10 Uchino T, Endo F, Ikeda S, et al. Three brothers with progressive hepatic dysfunction and severe steatosis due to patent ductus venosus. Gastroenterology 1996;110:1964-8. 


\section{URSO in obstetric cholestasis: not a bear market}

Obstetric cholestasis (synonym intrahepatic cholestasis of pregnancy) is rapidly emerging from the realms of clinical impressions into a scientific framework. Obstetricians, not least in Britain, have maintained a generally sceptical attitude towards attempts to recognise it as a significant clinical entity. Nevertheless, a consensus is emerging which acknowledges that obstetric cholestasis has major clinical implications for mother and baby. ${ }^{1}$ The pregnant woman may be driven to distraction by severe pruritus, most severely felt on hands and feet, which leads to regular cold baths and other ineffectual palliation during stressful sleepless nights. The brush off that "itching is of no consequence" and that "everyone itches in pregnancy" merely adds insult to injury. Mothers with a history of obstetric cholestasis have a higher incidence of gallstones. Babies are at increased risk of premature labour with fetal distress and there is a significantly increased risk of stillbirth. Traditional treatments for the pruritus of cholestasis are not very effective, and sequestrants such as cholestyramine exacerbate the tendency to vitamin $\mathrm{K}$ deficiency with its attendant risk of perinatal and post-partum haemorrhage.

Several recent reports have emerged of the efficacy of ursodeoxycholic acid (URSO), taken orally, in relieving both the pruritus and biochemical derangements associated with obstetric cholestasis. ${ }^{234}$ URSO is the dominant bile acid in the bear but a minority species in humans. It is more hydrophilic than the other primary and secondary human bile acids and protects against the membrane damaging toxicity of its more hydrophobic partners by displacing them from the hepatocyte and partially replacing them in the bile acid pool. Itching remits partially or entirely and serum biochemistry including aminotransferases and serum bile acids improve in the majority of patients during treatment with URSO. ${ }^{5}$ The pivotal double blind controlled clinical trial mounted in Chile, where the incidence of obstetric cholestasis is the highest in the world, was terminated prematurely when only 15 patients had been randomised and a baby was stillborn to a mother who proved to have taken placebo. ${ }^{6}$ Despite the small numbers involved the data showed significant benefit of URSO over placebo with respect to measures of aminotransferases and serum bile acids. Treatment with URSO tended to normalise the cholate:chenodeoxycholate ratio of serum bile acids, but failed to restore normal concentrations of $7 \alpha$-hydroxy-4-cholesten-3-one, a measure of de novo bile acid synthesis; this did, however, return to normal in the one placebo treated patient who spontaneously remitted, suggesting that URSO is reversing many of the consequences of obstetric cholestasis without necessarily correcting the underlying defect. Analysis of progesterone metabolites in serum and urine of patients with obstetric cholestasis showed that disulphates with a $3 \alpha$-hydroxy- $5 \alpha$ $(\mathrm{H})$ configuration were notably increased but glucuronide conjugates were not. ${ }^{78}$ An increased ratio of $3 \alpha: 3 \beta$ hydroxysteroids was closely related to obstetric cholestasis - it was present in all patients with obstetric cholestasis and a reduction in the $3 \alpha: 3 \beta$ hydroxysteroid ratio was observed in all seven "responders" to URSO and the one patient in the placebo group who went into spon- taneous remission but not in the non-responder among the eight treated with URSO. The easiest way to understand this would be to postulate that URSO was facilitating the secretion into bile of metabolites, particularly progesterone metabolites, which were being retained as a consequence of the cholestasis. Sulphation of these metabolises by the liver is a protective action in that it attenuates their toxicity and promotes their solubility in water and excretion from the urine. The availability of sulphate for conjugation is likely to be limiting under those circumstances. ${ }^{9}$ Several transporters have recently been characterised which are specifically located to the canalicular membrane and which actively transport bile acids (BSEP), organic anions (MRP2), and phospholipid (MDR3) into bile, allied with genetic defects which impair their function and account for several syndromes of familial intrahepatic cholestasis. ${ }^{10}$ Jacquemin et al recently described a family in which a child was born with severe neonatal cholestasis due to homozygous mutations in the human multidrug resistance 3 (MDR3) gene. ${ }^{11}$ The child's mother and several maternal and paternal aunts were found to have suffered from obstetric cholestasis and to be heterozygous for the MDR3 mutation. We can surmise therefore that similar genetic defects in canalicular bile transporters may account for obstetric cholestasis in other predisposed individuals. URSO could alleviate this problem by modulating nuclear transcription of transporter genes, delivering more transporters to the canalicular membrane or increasing the efficiency of transporter function within the membrane. An effect of URSO on stereospecific progesterone metabolism is not excluded. Given this increasingly compelling evidence regarding both clinical efficacy and underlying scientific rationale for use of URSO in the treatment of obstetric cholestasis, the paper by Rodrigues et al in this issue (see page 446) is important as it suggests that use of URSO in pregnancy is safe. Concentrations of cholic acid and URSO in meconium correlated with maternal serum concentrations although the proportion of URSO barely doubled in meconium compared with an increase from relatively trace amounts to $18 \%$ in maternal serum. Despite the increased proportion of URSO in meconium of infants whose mothers were treated with it, there was no increase in its potentially toxic metabolite lithocholic acid. However, although the same group has also previously shown in vitro that URSO restores placental bile acid transport towards normal in placental vesicles from pregnancies complicated by obstetric cholestasis, ${ }^{12}$ in the study published here there was no diminution of cholic acid in meconium in response to URSO, suggesting that the fetus lacks the means of excreting these bile acids once they have accumulated. The authors contend on this basis, reasonably in my view, that early treatment with URSO is indicated to prevent this accumulation in the fetus of cholate derived from maternal serum, which it is apparently unable to excrete during the remainder of pregnancy. The lack of any reports of adverse fetal reaction to URSO when used to treat obstetric cholestasis is further supported.

The Liver and Biliary Unit,

E ELIAS

3rd Floor, Nuffield House,

Queen Elizabeth Hospital,

Edgbaston,

Birmingham B15 2TH, UK

1 Fisk NM, Storey GNB. Foetal outcome in obstetric cholestasis. Br f Obstet Gynaecol 1988;95:1137-43. 
2 Palma J, Reyes H, Ribalta J, et al. Effects of ursodeoxycholic acid in patients with intrahepatic cholestasis of pregnancy. Hepatology 1992;15:1043-7.

3 Floreani A, Paternoster D, Grella V, et al. Ursodeoxycholic acid in intrahepatic cholestasis of pregnancy. Br f Obstet Gynaecol 1994;101:64-5.

4 Davies MH, da Silva RCMA, Jones SR, et al. Foetal mortality associated with cholestasis of pregnancy and the potential benefit of therapy with ursodeoxycholic acid. Gut 1995;37:580-4.

5 Brites D, Rodrigues CMP, Oliveira N, et al. Correction of maternal serum bile acid profile during ursodeoxycholic acid therapy cholestasis of pregnancy. F Hepatol 1998;28:91-8.

6 Palma J, Reyes H, Ribalta J, et al. Ursodeoxycholic acid in the treatment of cholestasis of pregnancy: a randomised, double blind study controlled with placebo. F Hepatol 1997;27:1022-8

7 Meng L-J, Reyes H, Axelson M, et al. Progesterone metabolises and bile acids in serum of patients with intrahepatic cholestasis of pregnancy: effect of ursodeoxycholic acid therapy. Hepatology 1997;26:1573-9.
8 Meng L-J, Reyes H, Palma J, et al. Effects of ursodeoxycholic acid on conjugated bile acids and progesterone metabolises in serum and urine of patients with intrahepatic cholestasis of pregnancy. $f$ Hepatol 1997;27: 1029-40.

9 Davies MH, Ngong JM, Yucesoy $M$, et al. The adverse influence of pregnancy upon sulphation: a clue to the pathogenesis of intrahepatic cholestasis of pregnancy. F Hepatol 1991;21:1127-34.

10 Jansen PL, Muller MM. Progressive familial intrahepatic cholestasis types 1, 2, and 3. Gut 1998;42:766-77.

11 Jacquemin E, Cresteil D, Manouvrier S, et al. Heterozygous non-sense mutation of the MDR3 gene in familial intrahepatic cholestasis of pregnancy. Lancet 1999;353:210-11.

12 Serran MA, Brites D, Larena MG, et al. Beneficial effect of ursodeoxycholic acid on alterations induced by cholestasis of pregnancy in bile acid transport across the human placenta. F Hepatol 1998;28:829-39. 